This is an electronic reprint of the original article. This reprint may differ from the original in pagination and typographic detail.

Author(s): Paakkari, Olli; Torppa, Minna; Kannas, Lasse; Paakkari, Leena

Title: $\quad$ Subjective health literacy : Development of a brief instrument for school-aged children

Year: $\quad 2016$

Version:

Please cite the original version:

Paakkari, O., Torppa, M., Kannas, L., \& Paakkari, L. (2016). Subjective health literacy : Development of a brief instrument for school-aged children. Scandinavian Journal of Public Health, 44(8), 751-757. https://doi.org/10.1177/1403494816669639

All material supplied via JYX is protected by copyright and other intellectual property rights, and duplication or sale of all or part of any of the repository collections is not permitted, except that material may be duplicated by you for your research use or educational purposes in electronic or print form. You must obtain permission for any other use. Electronic or print copies may not be offered, whether for sale or otherwise to anyone who is not an authorised user. 


\section{Subjective health literacy: development of a brief instrument for school-aged children}

Olli Paakkari, Minna Torppa, Lasse Kannas \&Leena Paakkari

University of Jyväskylä, Jyväskylä, Finland

\section{Abstract}

Aims: The present paper focuses on the measurement of health literacy $(\mathrm{HL})$, which is an important determinant of health and health behaviours. $\mathrm{HL}$ starts to develop in childhood and adolescence; hence there is a need for instruments to monitor $\mathrm{HL}$ among younger age groups. These instruments are still rare. The aim of the project reported here was therefore to develop a brief, multidimensional, theorybased instrument to measure subjective $\mathrm{HL}$ among school-aged children. Methods: The development of the instrument covered four phases: item generation based on a conceptual framework, a pilot study $(n=405)$, test-retest $(n=117)$, and construction of the instrument $(n=3853)$. All the samples were taken from Finnish $7^{\text {th }}$ and $9^{\text {th }}$ graders. Results: Initially, 65 items were generated, of which 32 items were selected for the pilot study. After item reduction, the instrument contained 16 items. The test-retest phase produced estimates of stability. In the final phase a 10-item instrument was constructed, referred to as Health Literacy for School-aged Children (HLSAC). The instrument exhibited a high Cronbach alpha (.93), and included two items from each of five predetermined theoretical components (theoretical knowledge, practical knowledge, critical thinking, self-awareness, citizenship). Conclusions: The iterative and validity driven development process made it possible to construct a brief multidimensional HLSAC instrument. Such instruments are suitable for large-scale studies, and for use with children and adolescents. Validation will require further testing for use in other countries.

Key words: health literacy, measurement, school-aged children, adolescent, subjective 


\section{INTRODUCTION}

According to a World Health Organization report [1] there is a health literacy (HL) crisis in Europe and beyond, due to a mismatch between individuals' competence capital and the increasingly complex requirements posed in taking care of one's health. According to a recent study, over $10 \%$ of Europeans have insufficient $\mathrm{HL}$, while almost $50 \%$ have limited (insufficient or problematic) $\mathrm{HL}$ [2]. Inadequate $\mathrm{HL}$ has consequences at both the individual and societal level. It has been reported as an independent risk factor for health [3], being associated for example with problems in interpreting health-related information, more frequent hospitalization, incorrect use of medicines, poorer health status, and increased mortality [4] - all this in addition to higher healthcare costs for society as a whole [5].

During childhood, a foundation is laid for many health behaviours, and for overall health and wellbeing. Moreover, $\mathrm{HL}$ itself starts to develop in childhood, and there is therefore a need to focus on $\mathrm{HL}$ among younger age groups. At the present time, a particularly important issue for children and adolescents is the complexity of the challenges faced in taking care of one's health. The modern environment exposes children and adolescents to many unhealthy behaviours, such as physical inactivity [6] or excessive gain of energy [7]. Increasingly, the Internet and other media expose children to information of varying quality [8], with media platforms for both positive and negative learning about health issues [9].

There are numerous contested conceptualizations of HL. The definitions vary from a fairly narrow focus on the reading, writing, and numeracy needed in a healthcare or medical context (i.e. functional health literacy) [10], toward a broader focus involving cognitive skills, social skills, and the motivation to promote and maintain health in general $[11,12]$. It seems unlikely that any definition of HL will work in every situation; thus any useful or meaningful definition will be highly dependent on the purpose and context of its use. This holds true also for measures of HL. In fact, in parallel with the lack of an agreed HL definition, there is no generally accepted view on how HL should be measured [13].

Just as there have been differences in $\mathrm{HL}$ conceptualizations, there have been varying measures of $\mathrm{HL}$. These have ranged from a narrow focus on functional skills, as with the Test of Functional Health 
Literacy in Adults (TOFHLA) [14], toward broader competence operationalizations, as with the Health Literacy Questionnaire (HLQ) [15]. Moreover, variation exists according to whether one is seeking to measure subjective (i.e. self-reported, perceived) HL, or objective (performance-based) HL. There has been a tendency to attach more value to the objective measures [16]. However, current subjective measures have succeeded in measuring comprehensive (i.e. multidimensional) HL, which is still not the case with many objective tests [17]. Kiechle et al. [13] go so far as to argue that subjective instruments 'could allow more efficient research for health literacy', on the grounds that they can be more easily applied to large samples [18]. For the most part, subjective measures focus on individuals' perceived competence, that is, their self-efficacy. Many of these measures are based on more than three decades of extensive research, showing a clear link between self-efficacy (i.e. perceived competence) and health behaviour [19]. According to Bandura [20] 'efficacy beliefs influence goals and aspirations' and 'the stronger the perceived self-efficacy, the higher the goals people set for themselves and the firmer their commitment to them'. If this is so, it would indeed indicate a need to study how people perceive their competence regarding health-related issues. All in all, one can say that it is crucial to select or develop an instrument that fits with one's purposes, whether that purpose involves focusing on a narrow or broader notion of $\mathrm{HL}$, or an objective or subjective means of measurement.

Currently, we are in need of reliable and valid $\mathrm{HL}$ instruments for different age groups and contexts. Despite an increasing interest in measuring $\mathrm{HL}$, tools for examining children's and adolescents' subjective $H L$ at the population level are still rare $[21,22]$. Here it should be noted that in many studies aimed at measuring adolescents' $\mathrm{HL}$, the instruments applied have not been validated for the target group [23]. Moreover, the majority of studies on adolescents' HL have focused purely on functional health literacy, highlighting the need for instruments based on a broader notion of HL. Brevity is also a consideration, since the instruments used should be brief enough to be reliably used with children. The length of the instrument becomes particularly important when the aim is to use it in more extensive surveys, involving an examination of the links between $\mathrm{HL}$ and other phenomena, such as health behaviour or perceived health among children. The aim of the present study was thus to develop a brief, comprehensive, and theory-based instrument for measuring subjective $\mathrm{HL}$ among school-aged children. 


\section{METHODS}

The development of the HL instrument comprised four main phases [24]: item generation, piloting, test-

retest, and construction of the instrument (Table 1).

Table 1. Overview of the $\mathrm{HL}$ instrument development process

Phase

Item reduction

1. Item generation

Contextualization, the five core components of HL (theoretical knowledge, practical knowledge, critical thinking, self-awareness, citizenship)

Reading and synthesis of relevant literature and existing instruments

Formulation of the items

65 items $\rightarrow 32$ items

Item selection for the pilot study (iterative process of evaluation and discussion by an expert group)

2. Pilot study ( $n=405,7^{\text {th }}$ and $9^{\text {th }}$ graders)

Data analysis: inspection of distributions, reliability analysis/internal

32 items $\rightarrow 16$ items consistency, confirmatory factor analysis, factor loadings and content of the items, examination of model goodness and sufficiency

Qualitative item analysis: four class-level discussions on the comprehensibility of the items, reformulation of three items

3. Test-retest ( $n=117,7^{\text {th }}$ and $9^{\text {th }}$ graders)

A two-week interval, the same pupils, structural equation modelling with one latent $\mathrm{HL}$ factor and with the five factors derived from the theoretical core components

16 items

4. Construction of a brief instrument $\left(n=3853,7^{\text {th }}\right.$ and $9^{\text {th }}$ graders) Data analysis: inspection of distributions, reliability analysis/internal 16 items $\rightarrow$ consistency, confirmatory factor analysis, factor loadings and content of 15 items $\rightarrow$ the items, examination of model goodness and sufficiency, regression 10 items analysis to predict the relationship between 10 - and 15 -item instruments, 10 -item test finalized

Item generation (1st phase)

Item generation involved two steps, namely the conceptualization and synthesizing of relevant literature, and the formulation of the items. In the first place, as suggested by Pleasant, McKinney, and 
Rikard [25] the instrument was explicitly developed on a testable conceptualization of HL, i.e. one which highlights the multidimensional nature of the concept. Due to an interest in assessing (i) multidimensional HL, in (ii) an educational setting, among (iii) school-aged children, Paakkari and Paakkari's [26] conceptualization of $\mathrm{HL}$ as a learning outcome was chosen to guide the construction of the items. Their conceptualization of $\mathrm{HL}$ and its components is based on an understanding that $\mathrm{HL}$ can be explicitly defined, operationalized, and translated into pedagogical practices. This is particularly important when the intention is to move beyond $\mathrm{HL}$ assessment towards how health literacy can be developed further among the target group. According to such a conceptualization, $\mathrm{HL}$ 'comprises a broad range of knowledge and competencies that people seek to encompass, evaluate, construct and use. Through health literacy competencies people become able to understand themselves, others and the world in a way that will enable them to make sound health decisions, and to work on and change the factors that constitute their own and others' health chances'. [26, 136]

This definition of HL incorporates five core components: theoretical knowledge, practical knowledge, individual critical thinking, self-awareness, and citizenship (Table 2). These components (in other words broader competence areas) are partly overlapping, and they are not necessarily fully hierarchical, even if some elements of a hierarchy can be found. The components encompass how HL expands from mere literacy on certain health topics towards a deeper literacy, in terms of realizing the potentials in oneself, others, and the world beyond. 
Table 2. The contextualization of HL: the core components [26]

\section{Five core components of $\mathrm{HL}$}

The theoretical knowledge of health issues contains a range of principles, theories, and conceptual models. Knowledge is viewed as something explicit, factual, universal, formal, and declarative. It includes lower levels of thinking skills, such as remembering.

Practical knowledge (i.e. procedural knowledge, skills) can be seen as a competency that allows one to put theoretical knowledge into practice. Whereas theoretical knowledge is something applicable to many different situations, practical knowledge can be seen as usable only in specific contexts. It is partly rooted in the individual's experiences, and thus it includes tacit, intuitive, or implicit knowledge. Practical knowledge contains basic health skills such as the ability to find health information, the ability to seek health services, and the ability to give first aid.

Individual critical thinking can be understood as the ability to think clearly and rationally. It is based on having a curious and investigative attitude towards the world, and a desire to understand health issues in a deeper way. In practice, critical thinking contains higher level thinking skills, such as an ability to analyse, evaluate, and create something new; this could include e.g. the ability to search for the logical connections between health ideas, to solve problems, to argue, to draw conclusions, or to assess the validity of health information.

Self-awareness is the ability to reflect on oneself and it make possible the personal contextualization of health issues. Through self-reflection, the individual becomes conscious of his/her own thoughts, feelings, needs, motives, values, attitudes, and experiences, and is able to consider how these relate to ways of behaving in an individually health-enhancing way. An important part of self-awareness is the ability to reflect on oneself as a learner.

Citizenship involves the ability to take social responsibility, and to think of the probable consequences of one's own actions on others. The ability to act in an ethically responsible way means that individuals are able to consider health issues beyond their own perspective: they may become aware of their own rights and responsibilities, and the effects our actions or thoughts may have on other people or on the environment. The component further includes the ability to identify and work on factors that influence one's own and other people's possibilities to achieve or maintain good health.

Thereafter, existing theoretical and empirical HL literature (and also relevant health promotion and educational literature) was critically examined by an expert group. The group consisted of researchers in the field of health promotion, education, and psychology. The group members were experienced in teaching at different levels of the educational system (comprehensive school, upper secondary school, and higher education), and in developing national curricula for health education in schools. There was also consultation with external experts in developing $\mathrm{HL}$ instruments. Previous studies on $\mathrm{HL}$ instrument development (such as HLQ [15] and the European Health Literacy Survey Questionnaire (HLS-EU-Q) [27]) afforded ideas, patterns, and examples for item design, as did Bloom's taxonomy [28]. The expert group 
aimed to ensure that the content of the items matched the core components of HL (content validity [29]).

The expert group then generated 65 items covering all five core components of HL. After an iterative process of reading and re-reading, discussion with the expert group, and consultation with external researchers, 32 items were included in the pilot study. The item formulation and the response scale were based on the guidelines for measuring general self-efficacy [30]. Thus, all the items took the form "I am confident that...", with the response options ranging from "not at all true" to "absolutely true" (Likert scale with 4 options). There were no non-committal options (such as "I can't say" or "No opinion"). In the instrument itself the items corresponding to a given component (out of the five) were mixed among items from the other components.

The pilot study (2nd phase)

In the autumn of 2013, the HL instrument with 32 items was piloted in two upper secondary schools in Finland. This formed part of a pilot study for the collaborative cross-national project of the World Health Organization (WHO) entitled Health Behaviour in School-aged Children (HBSC) [31]. The schools were chosen via a discretionary sampling method. The sample included a total of 401 pupils (7th graders, $n=202$; 9th graders, $n=199$ ). The pupils filled the survey anonymously in the course of one lesson (lasting 45 minutes).

In analysing the responses, the distributions of all the items were examined, and four items with very low discrimination power were removed. Next, a confirmatory factor analysis (CFA) was applied to the remaining 28 items (in conjunction with theoretical consideration of the item contents) to identify the best items. The model to which the CFA was applied was specified a priori, on the basis of a theoretical conceptualization involving five fixed factors. Items were removed one by one, on the basis of the factor loadings, plus careful consideration of the item content. 
The fit of the model to the data was tested via the following: Chi-square test, Root Mean Square Error of Approximation (RMSEA), Comparative fit Index (CFI), Tucker-Lewis Index (TLI), and Standardized Root Mean square Residual (SMRM). All the analyses were conducted with Mplus 7.3. Because the data had a hierarchical structure (pupils nested in classrooms), models were built with a COMPLEX option provided in Mplus; this was done to correct for the standard errors in the models according to the nested data structure.

With a view to content validity, all the items were discussed with adolescents, in order to secure maximum comprehensibility and clarity of the items. The discussions were conducted in four classes (two $7^{\text {th }}$ grade classes and two $9^{\text {th }}$ grade classes, with 20-24 pupils in each class).

Test-retest (3rd phase)

The version used in the test-retest phase consisted of 16 items. The sample consisted of 117 pupils ( $7^{\text {th }}$ and $9^{\text {th }}$ graders) from one school. The school was a normal Finnish upper secondary school, and it was selected via discretionary sampling. Fourteen pupils dropped out between the measurements. The testretest was organized under similar conditions, but with a two-week interval. There are differences in the guidelines for the optimum test-retest interval, but it has been argued that a shorter interval may help students to remember their answers, and that a longer interval between the measurements can indicate the stability of the phenomenon rather than the reliability of the instrument [24].

Response consistency over time [29] was examined via structural equation modelling (SEM), using Mplus 7.3. The latent HL factor for the 16 items was estimated for both time points. In the model, the retest latent factor was regressed on the latent $\mathrm{HL}$ factor at the first assessment time-point. No error covariances between the items were allowed in the models. In addition, the stability of the five theoretical components was examined by constructing the model with the same five latent factors at test and retest. 
Construction of a brief instrument (4th phase)

The data were collected in Finland in 2014, as part of the HBSC study. The HBSC study provides information on adolescents' demographic factors, health behaviours (health promoting behaviours, risk behaviours), perceived health, lifestyles, and life circumstances; thus the HL instrument (having reduced it to 16 items) was part of this broader survey. The nationally representative sample consisted in total of 3853 pupils (for $7^{\text {th }}$ graders $n=1918$; for $9^{\text {th }}$ graders $n=1935$ ) from 359 schools. The schools were chosen from the Finnish school register using a cluster sampling method that took into consideration provinces, the type of municipality (urban, semi-urban, rural), and the size of the schools. The participating class in each school was randomly selected. Thirteen- and fifteen-year-old participants responded voluntarily and anonymously to a standardized questionnaire administered in the course of one 45-minute lesson. Pupils were informed about the confidentiality of the study, and the fact that only group-level results would be reported.

As a first step, the instrument was reduced from 16 to 15 items, with each factor having an equal number of items. One extra item that had remained from the previous round (involving the selfawareness factor) was removed after inspection of the distributions and the item loadings. The 10-item version of the instrument was constructed using the same item reduction process as in the pilot study phase, i.e. inspection of the item distributions, internal consistency estimates (Cronbach's alpha), factor loadings, and CFA model fit, in addition to examination of the contents of the items. In order to examine the sufficiency of the 10-item version of the instrument, a regression analysis was conducted in which the 10-item version was set to predict the longer version of the instrument.

\section{RESULTS}

\section{Pilot study}

At the start of the pilot study there were 32 items, then after item reduction the instrument contained 16 items. The plan had been to reach a valid and reliable 15 -item solution with three items representing 
each theoretical HL component. However, one extra item was added to the self-awareness component, for content-based reasons, and with a view to finding the best possible combination of items.

The Cronbach alpha for the entire 16-item HL-measure was very high (.94). In the final one-factor CFA model with 16 items, no error covariances between the items were allowed $\left(\chi^{2}(104)=261.69, p=.000\right.$;

RMSEA $=.06, \mathrm{CFI}=.93, \mathrm{TLI}=.92, \mathrm{SRMR}=.04)$. The standardized factor loadings were between .57 and .74, with the majority being above .70 . The Cronbach alphas for each of the five factors (based on the theoretical $\mathrm{HL}$ components) were between .75 and .84 . Hence the internal consistency of each of the $\mathrm{HL}$ components was at an adequate level.

The pilot phase included also class-level discussions with the pupils. In general the pupils considered that the items were understandable. However, three items required some reformulation to avoid ambiguity.

\section{Test-retest}

The reformulated 16-item instrument was utilized with 117 pupils in the test-retest phase. In the SEM with one latent $\mathrm{HL}$ factor at both assessments, the standardized stability estimate was .83. The SEM with five factors also exhibited high stability estimates: theoretical knowledge .88 , practical knowledge .81 , critical thinking .81 , self-awareness .84 , and citizenship .90 . These estimates suggested adequate test-retest reliability for $\mathrm{HL}$, and also for the predetermined factors.

\section{Construction of the 10-item HLSAC instrument}

As an initial step, the instrument was reduced to 15 items, with each factor addressed by three items. Thereafter, the instrument was reduced to a 10-item Health Literacy for School-aged Children (HLSAC) instrument (figure 1). Five of the ten items were informed by the HLQ [15]. The distributions of all the 
items were reasonable, and none of the answer options accounted for more than $50 \%$ of the answers. For the 10-item instrument, Cronbach's alpha was high (.93), suggesting high internal consistency. The Cronbach alphas for the five core components (each with two items) were also reasonable (.69-.77).

Figure 1. The Health Literacy for School-aged Children (HLSAC) instrument: Cronbach alphas, item loadings, and $\mathrm{R}^{2}$ values
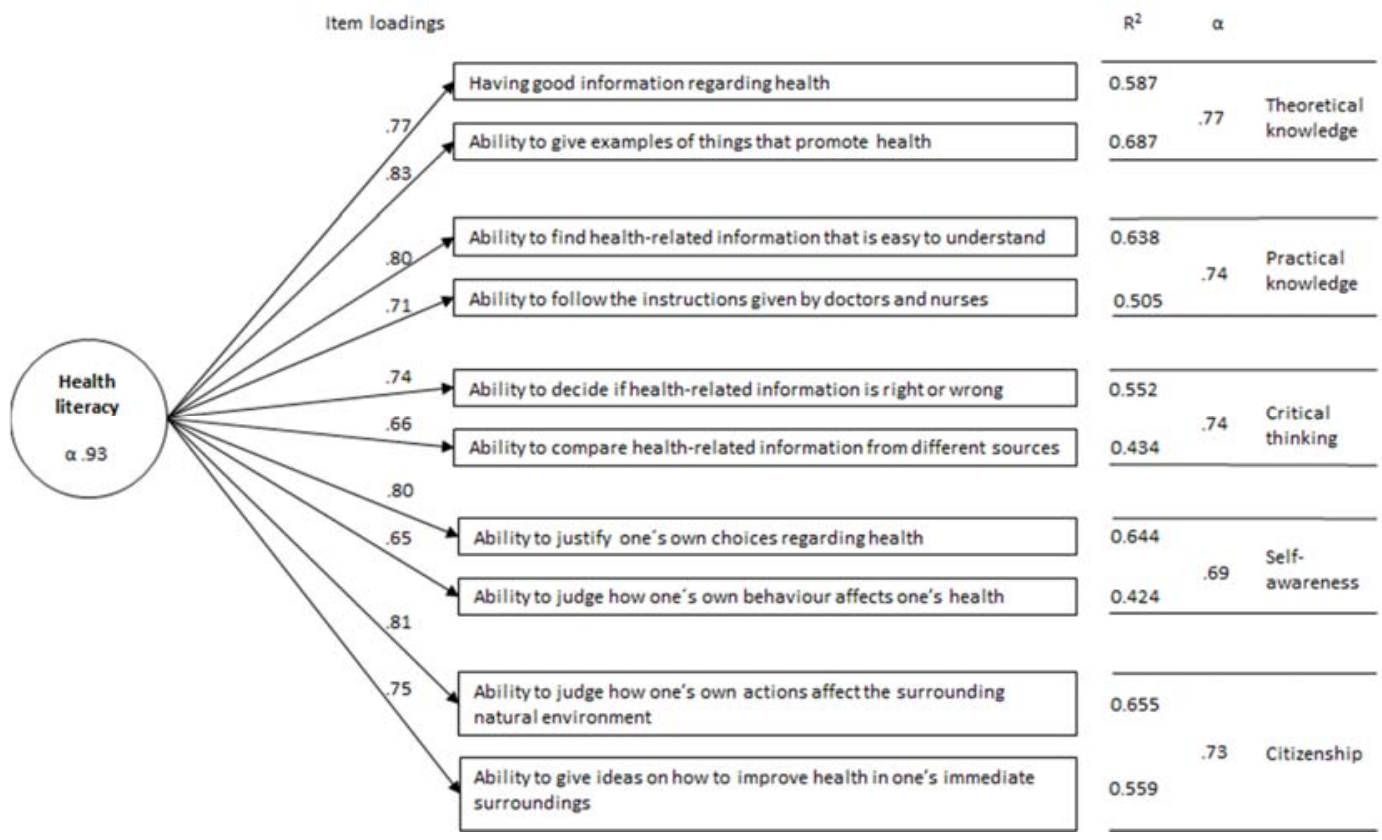

The 5-factor model with 10 items $\left(\chi^{2}(25)=681.41, p<.001 ;\right.$ RMSEA $=.08, \mathrm{CFI}=.96, \mathrm{TLI}=.92, \mathrm{SRMR}=$ .03) showed strong correlations (.95-1) between the factors. For this reason, the final model (figure 1) was constructed as a one-factor model. The model had good item loadings and - considering the large sample size and the fact that no error covariances were allowed between any of the items - it also had a reasonably good fit to the data $\left(\chi^{2}(35)=948.64, p<.001, \mathrm{RMSEA}=.08, \mathrm{CFI}=.94, \mathrm{TLI}=.92\right.$ and SRMR $=$ .04.). 
Finally, a regression analysis was conducted in order to examine the relationship between the 10- and the 15 -item instrument. The 10 -item HLSAC instrument predicted approximately $97 \%$ of the variance of the 15-item instrument $\left(R^{2}=.97, p<.001\right)$.

\section{DISCUSSION}

The objective of this study was to develop a brief and comprehensive theory-based instrument (HLSAC) to measure school-aged children's subjective HL. The instrument was based on a definition of HL [26], and was explicitly operationalized to measure five theoretical components. The goal of the iterative, systematic, and validity-driven development process [24] was that the instrument should encompass a wide range of competencies, covering the demands made on pupils by present and future society. In conjunction with this, bearing in mind that the theoretical background is based on seeing $\mathrm{HL}$ as a learning outcome, the items should be capable of being transformed into pedagogical practices, with a view to developing the competence that any given item is taken to represent.

There seems to be a clear need for this sort of instrument, given the lack of population-level instruments for inspecting school-aged children's subjective HL [21, 22]. Given that a low HL level seems to be a risk factor for health [3] and associated with a poorer health status [4], and also that the foundation for health is built during childhood, it is important to gain information on the overall level of children's HL, and further, on how the level of HL varies between different subgroups. It should then be possible to design and direct effective interventions. The availability of a brief, valid, and reliable instrument is important for large-scale studies, and is particularly useful in studies with children and adolescents. If the survey is too long, or if the instrument contains too many items, pupils get tired of responding, and this is likely to reduce the reliability of the study. Since the instrument we finally arrived at contains only ten items, it can be included in large scale surveys to measure the association between $\mathrm{HL}$ and other phenomena such as health behaviour or perceived health, without extending the overall length of the survey too far. 
The approach to developing an instrument followed generally accepted steps and principles. The major challenge was to determine items and combinations that represented wide theoretical core components; hence, the validity of the instrument was taken into account at every phase of study. Item generation was based on the iterative work of a broad, experienced, and multidisciplinary expert group, involving also external specialists. Discussions with adolescents on the comprehensibility of the items influenced the further formulation of the items. Moreover, findings from the pilot test and from the test-retest phase directed and contributed to the development of the final brief instrument. The results indicated that the short HLSAC instrument (i.e. ten items, with two items per theoretical core component) gives a good representation of the longer instrument. This is a significant issue when one has to consider how short an instrument can be without losing important aspects of HL (viewed as multidimensional in nature). The reliability of the instrument was found to be adequate. The high value for Cronbach's alpha indicated that the selected items all measured the same construct; furthermore, the test-retest of the instrument showed high consistency over time, in terms of encompassing one latent factor, and also the five preselected factors. According to the results, the overall goodness and adequacy of the instrument were clearly at an acceptable level.

Nevertheless, it is possible to identify limitations, and challenges for further development, even if the instrument itself worked well with the participants. There is a clear need now to test how far the instrument is applicable to younger pupils. Furthermore, given that $\mathrm{HL}$ is a complex phenomenon, there will inevitably be ongoing discussion of the essential $\mathrm{HL}$ components and their associations, of what a certain item may represent, and of how items should be formulated. Discussion may touch on the fact that in the model with 5 factors and 10 items there were correlations between the factors, with the final model thus being constructed as a one-factor model. The one-factor model describes a single phenomenon, here taken to denote 'health literacy'. It should be noted that on the basis of the background theory, the core components are partly overlapping and have some hierarchical elements; hence it is not surprising that cross-correlations were found. 
One significant issue involves comparing the results of subjective and objective HL measurements, and studying how the level of $\mathrm{HL}$ is connected to adolescents' self-reported health behaviour, or perceived health. Obtaining international comparative data could deepen our understanding of how well the HLSAC instrument works in different cultural and educational contexts. Overall, research on the measurement of adolescents' HL is very important, not just for the sake of making a methodological contribution to the field, but also for the health of the adolescents themselves.

\section{Declaration of conflicting interests}

None declared.

\section{Funding}

This work was supported by the National Institute for Health and Welfare, Finland. 


\section{References}

[1] Kickbusch I, Pelikan JM, Apfel F, et al. Health literacy: The solid facts. Copenhagen: World Health Organization 2013, p. 1.

[2] Sørensen K, Pelikan JM, Rothlin F, et al. Health literacy in Europe: comparative results of the European health literacy survey (HLS-EU). Eur J Public Health 2015; 25: 1053-1058.

[3] Volandes AE and Paasche-Orlow MK. Health literacy, health inequality and a just healthcare system. The Am J Bioeth 2007; 7: 5-10.

[4] Berkman ND, Sheridan SL, Donahue KE, et al. Low health literacy and health outcomes: an updated systematic review. Ann Int Med 2011; 155: 97-107.

[5] Howard DH, Gazmararian J and Parker RM. The impact of low health literacy on the medical costs of medicare managed care enrollees. Am J Med2005; 118: 371-377.

[6] Davison KK and Lawson CT. Do attributes in the physical environment influence children's physical activity? A review of the literature. Int J Behav Nutr Phys Act 2006; 3: 19.

[7] Patrick $\mathrm{H}$ and Nicklas TA. A review of family and social determinants of children's eating patterns and diet quality. J Amer Coll Nutr 2005; 24: 83-92.

[8] Mackey TK, Liang BA, Kohler JC, et al. Health domains for sale: the need for global health internet governance. J Med Internet res 2014; 16: e62.

[9] Begoray DL, Banister EM, Wharf Higgins J, et al. Puppets on a string? How young adolescents explore gender and health in advertising. JMLE 2015; 6: 57-64. 
[10] Parker RM, Baker DW, Williams MV, et al. The test of functional health literacy in adults. J Gen Inter Med 1995; 10: 537-541.

[11] Nutbeam D. Health promotion glossary. Health Promot Int 1998; 13: 349-364.

[12] Sørensen K, Van den Broucke S, Fullam J, et al. Health literacy and public health: a systematic review and integration of definitions and models. BMC Public Health 2012; 12: 80.

[13] Kiechle ES, Bailey SC, Hedlund LA, et al. Different Measures, Different Outcomes? A Systematic Review of Performance-Based versus Self-Reported Measures of Health Literacy and Numeracy. J Gen Intern Med 2015; 30: 1538-46.

[14] Baker DW, Williams MV, Parker RM, et al. Development of a brief test to measure functional health literacy. Patient Educ Couns 1999; 38: 33-42.

[15] Osborne RH, Batterham RW, Elsworth GR, et al. The grounded psychometric development and initial validation of the Health Literacy Questionnaire (HLQ). BMC Public Health 2013; 13: 658.

[16] McCormack L, Haun J, Sørensen K, et al. Recommendations for advancing health literacy measurement. J Health Commun 2013; 18: 9-14.

[17] Altin SV, Finke I, Kautz-Freimut S, et al. The evolution of health literacy assessment tools: a systematic review. BMC Public Health 2014; 14: 1207.

[18] Manganello JA, DeVellis RF, Davis TC, et al. Development of the Health Literacy Assessment Scale for Adolescents (HAS-A). J Comm Healthcare 2015; 8: 172-84. 
[19] Conner $\mathrm{M}$ and Norman P. Predicting health behavior: a social cognition approach. In: Conner M and Norman P (eds) Predicting health behaviour: research and practice with social cognition models. $2^{\text {nd }}$ ed. Maidenhead: Open University Press. 2005, pp. 1-27.

[20] Bandura A. Health promotion by social cognitive means. Health Educ Behav 2004; 31: 143-164.

[21] Haun JN, Valerio MA, McCormack LA, et al. Health literacy measurement: An inventory and descriptive summary of 51 instruments. J Health Commun 2014; 19: 302-333.

[22] Ormshaw M, Paakkari L and Kannas L. Measuring child and adolescent health literacy: a systematic review. Health Education 2013; 113: 433-455.

[23] Perry EL. Health literacy in adolescents: an integrative review. J Spec Pediatr Nurs 2014; 19: 210218.

[24] de Vet HCW, Terwee CB, Mokkink LB, et al. Measurement in medicine. Practical guides to biostatistics and epidemiology. Cambridge: Cambridge University Press, 2015: 30-32, 125.

[25] Pleasant A, McKinney J and Rikard RV. Health literacy measurement: a proposed research agenda. J Health Commun 2011; 16: 11-21.

[26] Paakkari L and Paakkari O. Health literacy as a learning outcome in schools. Health Education 2012; 112: $133-152$.

[27] Sørensen K, Van den Broucke S, Pelikan J, et al. Measuring health literacy in populations: illuminating the design and development process of the European Health Literacy Survey Questionnaire (HLS-EU-Q). BMC Public Health 2013; 13: 948. 
[28] Bloom BS. Taxonomy of educational objectives: the classification of educational goals. Handbook I: Cognitive domain. New York: McKay, 1956.

[29] Mokkink LB, Terwee CB, Patrick DL, et al. The COSMIN study reached international consensus on taxonomy, terminology, and definitions of measurement properties for health-related patient-reported outcomes. J Clin Epidemiol 2010; 63: 737-745.

[30] Schwarzer R and Fuchs R. Self-efficacy and health behaviour. In Conner M and Norman P (eds) Predicting health behavior. Buckingham: Open University Press, 1995, 163-196.

[31] Currie C, Nic Gabhainn S, Godeau E and the International HBSC Network Coordinating Committee. The Health Behaviour in School-aged Children: WHO Collaborative Cross-National (HBSC) Study: origins, concept, history and development 1982-2008. Int J Public Health 2009; 54: 131-139. 